\title{
Optical absorption of non-interacting tight-binding electrons in a Peierls-distorted chain at half band-filling
}

\author{
F. Gebhard* \\ ILL Grenoble, B. P. 156x, F-38042 Grenoble Cedex 9, France \\ K. Bott, M. Scheidler, P. Thomas, S. W. Koch \\ Dept. of Physics and Materials Sciences Center, \\ Philipps University Marburg, D-35032 Marburg, Germany \\ (to appear in The Philosophical Magazine B)
}

\begin{abstract}
In this first of three articles on the optical absorption of electrons in half-filled Peierls-distorted chains we present analytical results for non-interacting tightbinding electrons. We carefully derive explicit expressions for the current operator, the dipole transition matrix elements, and the optical absorption for electrons with a cosine dispersion relation of band width $W$ and dimerization parameter $\delta$. New correction (" $\eta$ "-)terms to the current operator are identified. A broad band-to-band transition is found in the frequency range $W \delta<\omega<W$ whose shape is determined by the joint density of states for the upper and lower Peierls subbands and the strong momentum dependence of the transition matrix elements.
\end{abstract}

PACS1996: 71.20.Rv, 71.10.Ca, 36.20.Kd

*e-mail: florian@gaston.ill.fr 


\section{INTRODUCTION}

Polymers and charge-transfer salts are examples for almost ideal one-dimensional materials (Farges 1994). Besides their potential technological applications these systems play an important test ground for our theoretical understanding of electron-electron and electronphonon interactions in low dimensions. The theoretical study of the corresponding onedimensional models instead of their three-dimensional counterparts has the further advantage that much larger systems can be handled numerically, and some of the conceptually simplest can even be solved analytically.

If one wants to show that a model is indeed appropriate for a real material one has to calculate experimentally accessible quantities. Analytical calculations often require the introduction of further (uncontrolled) approximations while the analysis of data from numerical approaches is hampered by finite size effects. This makes the assessment of the model quality a difficult task. Even if a model can be solved without resorting to uncontrolled approximations the interpretation and relevance of the results for real materials remains a matter of debate. Theoretical investigations concentrate on ideal systems, e.g., infinitely extended and perfectly homogeneous, that cannot be made in practice. Thus it should be kept in mind that experimental results may dominantly show effects of disorder and interactions with the chain environment which are usually neglected in theoretical approaches.

Despite these conceptual problems the analysis of basic models remains the first task to be accomplished in theory. In principle, models will differ in their experimental predictions which will allow to discriminate between them. In addition, model parameters are usually fitted by comparison with experiment.

It is widely believed that polyacetylene and other polymers are basically described by independent electrons coupled to a linear chain of atoms (Heeger, Kievelson, Schrieffer and Su 1988); (Baeriswyl, Campbell and Mazumdar 1992); (Schott and Nechtschein 1994). For a fixed lattice distortion the generic model is the dimerized tight-binding (or Hückel) model. The optical absorption for this independent free electron model was already addressed in 
the past (Baeriswyl, Harbeke, Kiess, Meier, and Meyer 1983); (Abe 1993). In this work we comprehensively derive analytical expressions for the dipole matrix elements and the optical absorption in the tight-binding approximation. In contrast to previous works we take special care of the derivation of the current and the dipole operator.

Some charge-transfer salts are understood as strongly correlated one-dimensional electron systems for half-filled bands (Mott-Hubbard insulators) (Alcácer, Brau, and Farges 1994). The extended Hubbard model for interacting electrons on a distorted chain at half-filling is considered appropriate for these materials (Mazumdar and Dixit 1986); (Fritsch and Ducasse 1991); (Mila 1995). The theoretical study of these model systems is subject to two forthcoming articles (Gebhard, Bott, Scheidler, Thomas, and Koch 1996).

The paper is organized as follows. In section \س we set up the Hamiltonian and diagonalize it. In section [II] we determine the current operator for tight-binding electrons on a Peierlsdistorted chain. We show that the distortion gives rise to correction terms that have been neglected in the literature. In section $[\nabla$ we derive the standard formulae for the optical conductivity. In section $\square$ we present explicit expressions for the optical absorption of free, independent tight-binding electrons moving on a Peierls-dimerized chain at half band-filling. In section $\nabla]$ we show how to derive the results via perturbation theory using the regular part of the electrical dipole operator. This approach is often preferred in semiconductor optics for the perturbational treatment of the external field. A summary closes our presentation.

\section{THE DIMERIZED TIGHT-BINDING HAMILTONIAN}

Free, non-interacting spin-1/2 electrons in a single band do not show optical absorption at finite frequencies because excitations with vanishing momentum necessarily have vanishing energy. All the optical conductivity is thus concentrated in the Drude weight at zero frequency. Hence, interactions must be included to obtain absorption at finite frequencies.

In highly anisotropic, quasi one-dimensional systems the lattice is unstable against a static (Peierls-)distortion at low temperatures since the gain in electron kinetic energy due to 
the distortion overcomes the lattice deformation energy. We assume that an electron transfer can take place between neighboring atoms only (tight-binding approximation). Furthermore, the wave vector of the lattice distortion, $q$, is taken commensurate with the electron filling, i.e., $q=2 k_{F}=\pi / a$ where $a$ is the lattice spacing of the undimerized chain. This implies that we address the half-filled case where the number of lattice sites $L$ equals the number of electrons, $N=N_{\uparrow}+N_{\downarrow}=L, n=N / L=1$.

Under these assumptions the electrons are described by a dimerized tight-binding model,

$$
\hat{T}(\delta)=-t \sum_{l=1, \sigma}^{L}\left(1+(-1)^{l} \delta\right)\left(\hat{c}_{l, \sigma}^{+} \hat{c}_{l+1, \sigma}+\hat{c}_{l+1, \sigma}^{+} \hat{c}_{l, \sigma}\right) .
$$

Here, $\hat{c}_{l, \sigma}^{+}\left(\hat{c}_{l, \sigma}\right)$ are the creation (annihilation) operators for an electron of spin $\sigma$ in a Wannier orbital centered at site $l .0 \leq \delta \leq 1$ describes the effect of the bond-length alternation on the electron transfer amplitudes. It implies $t=\left(t_{1}+t_{2}\right) / 2$, and $\delta=\left(t_{2}-t_{1}\right) /\left(t_{1}+t_{2}\right) \geq 0$ if one denotes the hopping amplitudes for the long (short) bond by $t_{1}\left(t_{2}\right)$. Usually, one supposes an exponential dependence of the electron transfer amplitudes on the distance $r$ between sites, $t(r)=t_{0} \exp (-(r-a) / \lambda)$. For $r_{1}=a(1+\eta / 2), r_{2}=a(1-\eta / 2)$ this implies $t_{0}=\sqrt{t_{1} t_{2}}$ and $\lambda=\eta a / \ln \left(t_{2} / t_{1}\right)$ where $\eta$ parametrizes the bond length alternation. Both $\delta$ and $\eta$ are usually small, e.g., $\delta=0.2$ and $\eta=-0.06$ are standard values for polyacetylene (Baeriswyl, Campbell, and Mazumdar 1992); (Schott and Nechtschein 1994). Note that $\delta$ and $\eta$ are always opposite in sign.

As usual the Hamiltonian can be diagonalized in momentum space. We apply periodic boundary conditions, and introduce the Fourier transformed electron operators as $\hat{c}_{k, \sigma}^{+}=$ $\sqrt{1 / L} \sum_{l=1}^{L} \exp (i k l a) \hat{c}_{l, \sigma}^{+}$for the $L$ momenta $k=2 \pi m /(L a), m=-(L / 2), \ldots(L / 2)-1$. We may thus write

$$
\hat{T}(\delta)=\sum_{|k| \leq \pi /(2 a), \sigma} \epsilon(k)\left(\hat{c}_{k, \sigma}^{+} \hat{c}_{k, \sigma}-\hat{c}_{k+\pi / a, \sigma}^{+} \hat{c}_{k+\pi / a, \sigma}\right)-i \Delta(k)\left(\hat{c}_{k+\pi / a, \sigma}^{+} \hat{c}_{k, \sigma}-\hat{c}_{k, \sigma}^{+} \hat{c}_{k+\pi / a, \sigma}\right)
$$

with the dispersion relation $\epsilon(k)$ and hybridization function $\Delta(k)$ defined as

$$
\begin{gathered}
\epsilon(k)=-2 t \cos (k a) \\
\Delta(k)=2 t \delta \sin (k a) .
\end{gathered}
$$


The Hamiltonian can easily be diagonalized in $k$-space by introducing the new Fermion quasi-particle operators $\hat{a}_{k, \sigma, \pm}^{+}$for these two bands which are related to the original electron operators by

$$
\begin{aligned}
& \hat{a}_{k, \sigma,-}=\alpha_{k} \hat{c}_{k, \sigma}+i \beta_{k} \hat{c}_{k+\pi / a, \sigma} \\
& \hat{a}_{k, \sigma,+}=\beta_{k} \hat{c}_{k, \sigma}-i \alpha_{k} \hat{c}_{k+\pi / a, \sigma}
\end{aligned}
$$

for $|k| \leq \pi /(2 a)$. The inverse transformation reads

$$
\begin{gathered}
\hat{c}_{k, \sigma}=\alpha_{k} \hat{a}_{k, \sigma,-}+\beta_{k} \hat{a}_{k, \sigma,+} \\
\hat{c}_{k+\pi / a, \sigma}=-i \beta_{k} \hat{a}_{k, \sigma,-}+i \alpha_{k} \hat{a}_{k, \sigma,+},
\end{gathered}
$$

where $\alpha_{k}^{2}+\beta_{k}^{2}=1$ has to be fulfilled for a canonical transformation. We note the helpful relations

$$
\begin{aligned}
\hat{c}_{k, \sigma}^{+} \hat{c}_{k, \sigma}-\hat{c}_{k+\pi / a, \sigma}^{+} \hat{c}_{k+\pi / a, \sigma}= & \left(\alpha_{k}^{2}-\beta_{k}^{2}\right)\left(\hat{a}_{k, \sigma,-}^{+} \hat{a}_{k, \sigma,-}-\hat{a}_{k, \sigma,+}^{+} \hat{a}_{k, \sigma,+}\right) \\
& +2 \alpha_{k} \beta_{k}\left(\hat{a}_{k, \sigma,-}^{+} \hat{a}_{k, \sigma,+}+\hat{a}_{k, \sigma,+}^{+} \hat{a}_{k, \sigma,-}\right) \\
\hat{c}_{k, \sigma}^{+} \hat{c}_{k, \sigma}+\hat{c}_{k+\pi / a, \sigma}^{+} \hat{c}_{k+\pi / a, \sigma}= & \hat{a}_{k, \sigma,-}^{+} \hat{a}_{k, \sigma,-}+\hat{a}_{k, \sigma,+}^{+} \hat{a}_{k, \sigma,+} \\
\hat{c}_{k, \sigma}^{+} \hat{c}_{k+\pi / a, \sigma}-\hat{c}_{k+\pi / a, \sigma}^{+} \hat{c}_{k, \sigma}= & i\left(\alpha_{k}^{2}-\beta_{k}^{2}\right)\left(\hat{a}_{k, \sigma,+}^{+} \hat{a}_{k, \sigma,-}+\hat{a}_{k, \sigma,-}^{+} \hat{a}_{k, \sigma,+}\right) \\
& +i 2 \alpha_{k} \beta_{k}\left(\hat{a}_{k, \sigma,+}^{+} \hat{a}_{k, \sigma,+}-\hat{a}_{k, \sigma,-}^{+} \hat{a}_{k, \sigma,-}\right) \\
\hat{c}_{k, \sigma}^{+} \hat{c}_{k+\pi / a, \sigma}+\hat{c}_{k+\pi / a, \sigma}^{+} \hat{c}_{k, \sigma}= & -i\left(\hat{a}_{k, \sigma,+}^{+} \hat{a}_{k, \sigma,-}-\hat{a}_{k, \sigma,-}^{+} \hat{a}_{k, \sigma,+}\right) .
\end{aligned}
$$

They allow to write the Hamiltonian in momentum space, eq. (2), as

$$
\begin{array}{r}
\hat{T}(\delta)=\sum_{|k| \leq \pi /(2 a), \sigma}\left(\hat{a}_{k, \sigma,+}^{+} \hat{a}_{k, \sigma,+}-\hat{a}_{k, \sigma,-}^{+} \hat{a}_{k, \sigma,-}\right)\left[-\epsilon(k)\left(\alpha_{k}^{2}-\beta_{k}^{2}\right)-\Delta(k) 2 \alpha_{k} \beta_{k}\right] \\
+\left(\hat{a}_{k, \sigma,+}^{+} \hat{a}_{k, \sigma,-}+\hat{a}_{k, \sigma,-}^{+} \hat{a}_{k, \sigma,+}\right)\left[\epsilon(k) 2 \alpha_{k} \beta_{k}-\Delta(k)\left(\alpha_{k}^{2}-\beta_{k}^{2}\right)\right] .
\end{array}
$$

We demand that the mixing terms vanish. This gives the following relation between $\alpha_{k}=$ $\cos \phi_{k}$ and $\beta_{k}=\sin \phi_{k}$ 


$$
\tan \left(2 \phi_{k}\right)=\frac{\Delta(k)}{\epsilon(k)} .
$$

The mixing amplitudes become

$$
\begin{aligned}
2 \alpha_{k} \beta_{k} & =-\frac{\Delta(k)}{E(k)} \\
\alpha_{k}^{2}-\beta_{k}^{2} & =-\frac{\epsilon(k)}{E(k)} \\
\alpha_{k} & =\sqrt{\frac{1}{2}\left(1-\frac{\epsilon(k)}{E(k)}\right)} \\
\beta_{k} & =-\sqrt{\frac{1}{2}\left(1+\frac{\epsilon(k)}{E(k)}\right)} \operatorname{sgn}(\Delta(k))
\end{aligned}
$$

where $\operatorname{sgn}(x)$ is the sign function. Note that $\alpha_{k+\pi / a}=-i \beta_{k}, \beta_{k+\pi / a}=i \alpha_{k}$. This implies that $\alpha_{k+q}$ is complex, in general.

It is easily seen that the Hamiltonian is diagonal in the new operators. The result is

$$
\hat{T}(\delta)=\sum_{|k| \leq \pi /(2 a), \sigma} E(k)\left(\hat{a}_{k, \sigma,+}^{+} \hat{a}_{k, \sigma,+}-\hat{a}_{k, \sigma,-}^{+} \hat{a}_{k, \sigma,-}\right)
$$

Here, $\pm E(k)$ is the dispersion relation for the upper $(+)$ and lower $(-)$ Peierls band,

$$
E(k)=\sqrt{\epsilon(k)^{2}+\Delta(k)^{2}} .
$$

The dispersion of both bands in the reduced zone scheme is shown in figure 1. The band width is $W=4 t$. The model describes a Peierls insulator at half-filling because all states in the lower Peierls band are filled, all states in the upper Peierls band are empty, and there is the Peierls gap $\Delta^{\mathrm{P}}=W \delta$ between the two bands. Optical transitions at finite frequency are now possible in the frequency range $\Delta^{\mathrm{P}} \leq \hbar \omega \leq W$. We set $\hbar=1$ from now on.

\section{CURRENT OPERATOR AND ELECTRICAL CONDUCTIVITY}

To describe optical transitions we need to include the interaction with an external electrical field which is only slowly varying in space. Only a frequency dependent field will be 
considered in the following. In general, a one-dimensional system shall be described by a time-independent Hamilton operator. Optical excitations are created by the time-dependent perturbation (Maldague 1977); (Mahan 1990)

$$
\hat{H}_{\mathrm{int}}=-\hat{\jmath} \cdot \frac{\mathcal{A}(t)}{c}
$$

which couples the external vector potential $\mathcal{A}(t)$ to the current operator $\hat{\jmath}$ of the system $(c$ is the speed of light).

It is not trivial to derive the current operator for a dimerized system. We choose the Coulomb gauge and set the scalar potential to zero. Then the electrical field $\mathcal{E}(t)$ is given by the vector potential $\mathcal{A}(t)$ alone, $\mathcal{E}(t)=-(1 / c)(\partial \mathcal{A}(t)) /(\partial t)$, or, upon Fourier transformation,

$$
f(\omega)=\int_{-\infty}^{\infty} d t e^{i \omega t} f(t) \quad ; \quad f(t)=\int_{-\infty}^{\infty} \frac{d \omega}{2 \pi} e^{-i \omega t} f(\omega)
$$

$\mathcal{A}(\omega) / c=\mathcal{E}(\omega) /(i \omega)$. Both fields are real functions of time, so that they both fulfill $f(\omega)=$ $f^{*}(-\omega), f=\mathcal{A}, \mathcal{E}$. The electrical field can be taken constant over lattice distances since we are interested in optical excitations in the regime of electron volts. These energies are small compared to atomic energies, and the corresponding wave vectors are small compared to typical electron momenta.

The presence of the vector potential changes the amplitudes for the electron transfer between neighboring unit cells. In the tight-binding approximation they are given by

$$
t_{l+1, l}(\mathcal{A})=\int d \mathbf{x} \Phi_{l+1}^{*}(\mathbf{x})\left[\frac{1}{2 m}\left(\mathbf{p}+\frac{e}{c} \mathcal{A}\right)^{2}+V_{\text {atom }}(\mathbf{x})\right] \Phi_{l}(\mathbf{x})
$$

where $(-e)$ is the charge of an electron, and the term in square brackets is the band structure Hamiltonian for a single electron. $\Phi_{l}(\mathbf{x})$ are the Wannier functions centered at site $l$. We can apply a gauge transformation to formally eliminate the vector potential from the band structure Hamiltonian. The Wannier functions now acquire a field dependence,

$$
\Phi_{l}(\mathbf{x}) \mapsto \widetilde{\Phi}_{l}(\mathbf{x})=\left[\exp \left(i(e / c) \int^{\mathbf{R}_{l}} \mathcal{A}\left(\mathbf{x}^{\prime}\right) d \mathbf{x}^{\prime}\right)\right] \Phi_{l}(\mathbf{x})
$$

where $\mathbf{R}_{l}$ is the space coordinate of site $l$. Since the vector potential is independent of the position in the unit cell we may take the field dependence outside the integral for the transfer matrix elements. Now that the vector field is along the chain we can write 


$$
t_{l+1, l}(\mathcal{A})=\exp \left(-i e\left(R_{l+1}-R_{l}\right) \mathcal{A} / c\right) t_{l+1, l}
$$

This is the well-known Peierls substitution.

Note that we tacitly assumed that the Wannier wave functions remain undistorted albeit the atoms come closer to or farther away from each other due to the distortion. This implies $\left|R_{l+1}-R_{l}-a\right| \ll a$ as a requirement for the validity of the tight-binding approximation. Otherwise the atomic wave functions will be deformed which will ultimately result in a biatomic, molecular structure. In a dimerized system the distances between neighboring sites are given by

$$
R_{l+1}-R_{l}=a\left(1+\eta(-1)^{l}\right) \quad ; \quad R_{l}=a\left(l-(-1)^{l} \eta / 2\right)
$$

where $\eta$ is the relative distortion. Formally, we may allow $|\eta| \leq 1$ to check our results. On physical grounds we have to require $|\eta| \ll 1$ to be consistent with the tight-binding approximation. The small value $\eta \approx-0.06$ in polyacetylen (Baeriswyl, Campbell, and Mazumdar 1992); (Schott and Nechtschein 1994) indicates that in real materials the corrections due to the $\eta$-terms will dominate those due to Wannier function deformations. The latter corrections should be exponentially small for small $|\eta|$-values since the wave functions themselves decay exponentially as function of distance from the lattice site.

The Hamiltonian depends on the external field via the field dependent hopping amplitudes. We expand it in powers of the (weak) external vector potential and obtain

$$
\hat{T}(\delta, \mathcal{A})=\hat{T}(\delta)-\hat{\jmath} \frac{\mathcal{A}}{c}+\frac{e^{2} a^{2}}{2} \hat{T}^{\prime}(\delta, \eta)\left(\frac{\mathcal{A}}{c}\right)^{2}+\ldots
$$

which allows us to identify the operator for the paramagnetic particle current, $\hat{\jmath}$, and the operator for the diamagnetic field current, $\hat{T}^{\prime}(\delta, \eta)$, as

$$
\begin{aligned}
\hat{\jmath} & =(-e) \sum_{l, \sigma}(i t a)\left(\hat{c}_{l+1, \sigma}^{+} \hat{c}_{l, \sigma}-\hat{c}_{l, \sigma}^{+} \hat{c}_{l+1, \sigma}\right)\left(1+(-1)^{l} \delta\right)\left(1+(-1)^{l} \eta\right) \\
\hat{T}^{\prime}(\delta, \eta) & =(-t) \sum_{l, \sigma}\left(\hat{c}_{l+1, \sigma}^{+} \hat{c}_{l, \sigma}+\hat{c}_{l, \sigma}^{+} \hat{c}_{l+1, \sigma}\right)\left(1+(-1)^{l} \delta\right)\left(1+(-1)^{l} \eta\right)^{2} .
\end{aligned}
$$

The operator for the time-dependent perturbation theory in the external field is then identified as $\hat{H}_{\mathrm{int}}(t)=-\hat{\jmath} \cdot \mathcal{A}(t) / c$ which is the standard form, equation (12). The geometrical 
distortion increases (decreases) the electron tunneling between neighboring atoms ( $\delta$-terms) but at the same time decreases (increases) the size of the effective dipole ( $\eta$-terms).

The operator for the total current, $\hat{\jmath}_{\text {tot }}$, is not only given by $\hat{\jmath}$ because this quantity is not gauge invariant. In fact, the particle current is supplemented by the field current since the field itself carries momentum. We write

$$
\hat{\jmath}_{\text {tot }}=\hat{\jmath}+\hat{X} \mathcal{A} / c
$$

and demand that this quantity be gauge invariant. This fixes the unknown operator $\hat{X}$. Applying the same gauge transformation as above we can gauge away the term proportional to $\hat{X}$,

$$
\hat{\jmath}_{\text {tot }} \mapsto \hat{\jmath}(\mathcal{A})
$$

We expand eq. (20) to first order in $\mathcal{A}$ and find from eq. (21) that $\hat{X}=e^{2} a^{2} \hat{T}^{\prime}(\delta, \eta)$, as expected.

\section{OPTICAL CONDUCTIVITY}

The dielectric function $\widetilde{\epsilon}(\omega)$ and the coefficient for the linear optical absorption $\widetilde{\alpha}(\omega)$ are given by (Haug and Koch 1990)

$$
\begin{aligned}
\widetilde{\epsilon}(\omega) & =1+\frac{4 \pi i \sigma(\omega)}{\omega} \\
\widetilde{\alpha}(\omega) & =\frac{4 \pi \operatorname{Re}\{\sigma(\omega)\}}{n_{b} c}
\end{aligned}
$$

where $\operatorname{Re}\{\ldots\}$ denotes the real part and $n_{b}$ is the background refractive index. It is supposed to be frequency independent near a resonance. Hence, the real part of the optical conductivity directly gives the absorption spectrum of the system.

Standard time-dependent perturbation theory gives the Kubo formula for the optical conductivity (Maldague 1977); (Mahan 1990). The Fourier transform of the total current density and the Fourier transform of the electrical field $\mathcal{E}(\omega)$ define the optical conductivity 


$$
\sigma(\omega)=\frac{\mathcal{N}_{\perp}}{L a} \frac{\left\langle\hat{\jmath}_{\text {tot }}(\omega)\right\rangle}{\mathcal{E}(\omega)}=\frac{\chi(\omega)+\mathcal{N}_{\perp} e^{2} a\left\langle\hat{T}^{\prime}(\delta, \eta)\right\rangle / L}{i \omega}
$$

where $\langle\ldots\rangle$ means the expectation value in the ground state without the perturbation, and $\mathcal{N}_{\perp}$ is the number of chains per unit area perpendicular to the chain direction. Here, $\chi(\omega)$ is the current-current correlation function defined by

$$
\chi(\omega)=\frac{\mathcal{N}_{\perp}}{L a} i \int_{0}^{\infty} d t e^{i \omega t}\left\langle[\hat{\jmath}(t), \hat{\jmath}]_{-}\right\rangle
$$

where $\hat{\jmath}(t)$ is the Heisenberg current operator for the unperturbed system.

The current-current correlation function can be spectrally decomposed in terms of exact eigenstates $|n\rangle$ (energy $E_{n}$ ) of the unperturbed system as

$$
\chi(\omega)=\frac{\mathcal{N}_{\perp}}{L a} \sum_{n}|\langle 0|\hat{\jmath}| n\rangle|^{2}\left[\frac{1}{\omega+\left(E_{n}-E_{0}\right)+i \gamma}-\frac{1}{\omega-\left(E_{n}-E_{0}\right)+i \gamma}\right] .
$$

Although $\gamma=0^{+}$is infinitesimal we may introduce $\gamma>0$ as a phenomenological broadening

of the resonances at $\omega= \pm\left(E_{n}-E_{0}\right)$. The real part of the optical conductivity determines the absorption spectrum. It is given by

$$
\operatorname{Re}\{\sigma(\omega)\}=\frac{\operatorname{Im}\{\chi(\omega)\}}{\omega}=\frac{\mathcal{N}_{\perp} \pi}{L a \omega} \sum_{n}|\langle 0|\hat{\jmath}| n\rangle|^{2}\left[\delta\left(\omega-\left(E_{n}-E_{0}\right)\right)-\delta\left(\omega+\left(E_{n}-E_{0}\right)\right)\right]
$$

which is positive for all $\omega$.

\section{OPTICAL ABSORPTION IN PEIERLS INSULATORS}

According to equation (26) we have to calculate the transition matrix elements $|\langle n|\hat{\jmath}| 0\rangle|^{2}$ between the ground state $|0\rangle$ (energy $E_{0}$ ) and all exact excited states $|n\rangle$ (energy $E_{n}$ ). This is easily accomplished for non-interacting electrons.

The current operator in momentum space reads

$$
\begin{aligned}
\hat{\jmath} & =-e \sum_{l, \sigma} i t a\left(1+(-1)^{l} \delta\right)\left(1+(-1)^{l} \eta\right)\left(\hat{c}_{l+1, \sigma}^{+} \hat{c}_{l, \sigma}-\hat{c}_{l, \sigma}^{+} \hat{c}_{l+1, \sigma}\right) \\
& =-e\left[\sum_{|k| \leq \pi / a, \sigma}(1+\eta \delta) \frac{\partial \epsilon(k)}{\partial k} \hat{c}_{k, \sigma}^{+} \hat{c}_{k, \sigma}-i\left(1+\frac{\eta}{\delta}\right) \frac{\partial \Delta(k)}{\partial k} \hat{c}_{k+\pi / a, \sigma}^{+} \hat{c}_{k, \sigma}\right] .
\end{aligned}
$$


It is seen that the current cannot simply be derived from the $k$-derivative of the Hamiltonian $\hat{T}(\delta)$ in momentum space, eq. (2). There are additional terms proportional to $\eta$ due to the fact that the distance between two lattice points is $\Delta r=a(1 \pm \eta / 2)$. This has been ignored in previous treatments (Genkin and Mednis 1968); (Cojan, Agrawal, and Flytzanis 1977); (Baeriswyl, Harbeke, Kiess, Meier, and Meyer 1983); (Abe 1993).

In terms of the new Fermions the current operator can be split into two terms, one which acts within the Peierls subbands while the other induces transitions between them, $\hat{\jmath}=\hat{\jmath}_{\text {intra }}^{\mathrm{P}}+\hat{\jmath}_{\text {inter }}^{\mathrm{P}}$. We obtain

$$
\begin{aligned}
& \hat{\jmath}_{\text {inter }}^{\mathrm{P}}=\sum_{|k| \leq \pi /(2 a), \sigma} \lambda_{\text {inter }}^{\mathrm{P}}(k)\left(\hat{a}_{k, \sigma,+}^{+} \hat{a}_{k, \sigma,-}+\hat{a}_{k, \sigma,-}^{+} \hat{a}_{k, \sigma,+}\right) \\
& \hat{\jmath}_{\text {intra }}^{\mathrm{P}}=\sum_{|k| \leq \pi /(2 a), \sigma} \lambda_{\text {intra }}^{\mathrm{P}}(k)\left(\hat{a}_{k, \sigma,-}^{+} \hat{a}_{k, \sigma,-}-\hat{a}_{k, \sigma,+}^{+} \hat{a}_{k, \sigma,+}\right)
\end{aligned}
$$

with the transition matrix elements

$$
\begin{aligned}
& \lambda_{\text {inter }}^{\mathrm{P}}(k)=e a\left[\delta \frac{(2 t)^{2}}{E(k)}+\eta E(k)\right] \\
& \lambda_{\text {intra }}^{\mathrm{P}}(k)=e a\left(1-\delta^{2}\right) \frac{\Delta(k) \epsilon(k)}{\delta E(k)} .
\end{aligned}
$$

If we restrict ourselves to half-filling or, more generally, to transitions for positive frequencies (interband transitions) we may ignore intraband transitions which only contribute to the optical conductivity $\sigma(\omega)$ at $\omega=0$.

Now it is very simple to calculate the optical absorption with the help of the spectral decomposition after the Hamiltonian has been diagonalized. One obtains

$$
\begin{aligned}
\operatorname{Re}\{\sigma(\omega>0)\} & =\frac{\pi \mathcal{N}_{\perp}}{L a \omega} \sum_{|k| \leq \pi /(2 a), \sigma}\left[\lambda_{\text {inter }}(k)\right]^{2} \delta(\omega-2 E(k)) \\
& =\frac{\mathcal{N}_{\perp} e^{2} a}{2 \omega^{2}} \frac{\left(\delta W^{2}+\eta \omega^{2}\right)^{2}}{\sqrt{\left(\omega^{2}-(W \delta)^{2}\right)\left(W^{2}-\omega^{2}\right)}} \text { for } \quad W \delta<\omega<W
\end{aligned}
$$

where $W=4 t$ is the band width, and $\mathcal{N}_{\perp}$ is the number of chains per unit area perpendicular to the chain direction. 
The optical absorption after eq. (29) is shown in figure 2. We plot the dimensionless reduced optical conductivity

$$
\sigma_{\text {red }}(\omega>0)=\frac{\omega \operatorname{Re}\{\sigma(\omega>0)\}}{\mathcal{N}_{\perp} a e^{2} W} .
$$

Furthermore we replace the energy conservation $\delta(\omega-2 E(k))$ by the smeared function

$$
\widetilde{\delta}(x)=\frac{\gamma}{\pi\left(x^{2}+\gamma^{2}\right)}
$$

to include effects of phonons and experimental resolution. This is equivalent to the replacement $\omega \rightarrow \omega+i \gamma$ in the formulae for the spectral decomposition of the optical conductivity.

The optical absorption $\widetilde{\alpha}(\omega)$ is not only given by the joint density of states for the upper and lower Peierls band but also reflects the strong momentum dependence of the interband matrix element $\lambda_{\text {inter }}(k)$. The contribution of the van-Hove singularity at $\omega=\delta W$ is thus enhanced compared to the singularity at $\omega=W$. This is the well-known "Umklapp enhancement" (Philipps 1966). The effect enforced by the contribution of the $\eta$-dependent terms which have not been taken into account in previous works (Genkin and Mednis 1968); (Cojan, Agrawal, and Flytzanis 1977); (Baeriswyl, Harbeke, Kiess, Meier, and Meyer 1983); (Abe 1993).

\section{ELECTRICAL DIPOLE OPERATOR}

In semiconductor physics (Haug and Koch 1990) one often prefers to calculate the optical susceptibility $\chi^{\mathrm{opt}}(\omega)$ which is related to the dielectric function via

$$
\widetilde{\epsilon}(\omega)=1+4 \pi \chi^{\mathrm{opt}}(\omega)
$$

Using eq. (22a) and (23) we find that

$$
\operatorname{Im}\left\{\chi^{\mathrm{opt}}(\omega)\right\}=\frac{\operatorname{Im}\{\chi(\omega)\}}{\omega^{2}}=\frac{\mathcal{N}_{\perp} \pi}{L a} \sum_{n} \frac{|\langle 0|\hat{\jmath}| n\rangle|^{2}}{\omega^{2}}\left[\delta\left(\omega-\left(E_{n}-E_{0}\right)\right)-\delta\left(\omega+\left(E_{n}-E_{0}\right)\right)\right] .
$$

We define the dipole operator $\hat{P}$ via 


$$
\hat{\jmath}=-i[\hat{P}, \hat{H}]_{-}
$$

and insert this definition into eq. (33). This allows us to write

$$
\operatorname{Im}\left\{\chi^{\mathrm{opt}}(\omega)\right\}=\frac{\mathcal{N}_{\perp} \pi}{L a} \sum_{n}|\langle 0|\hat{P}| n\rangle|^{2}\left[\delta\left(\omega-\left(E_{n}-E_{0}\right)\right)-\delta\left(\omega+\left(E_{n}-E_{0}\right)\right)\right]
$$

which is the result for the optical absorption calculated with the time-dependent perturbation operator

$$
\hat{H}_{\text {int }}(t)=-\hat{P} \mathcal{E}(t)
$$

In principle we could have used this operator for the time-dependent perturbation theory.

We can employ eq. (34) to identify the dipole operator. One finds

$$
\hat{P}=-e a \sum_{l, \sigma}\left(l-(-1)^{l} \eta / 2\right) \hat{c}_{l, \sigma}^{+} \hat{c}_{l, \sigma}=-e \sum_{l, \sigma} R_{l} \hat{c}_{l, \sigma}^{+} \hat{c}_{l, \sigma}
$$

which is indeed the result for the dipole operator in second quantization for electrons at positions $R_{l}$. Unfortunately, this operator is sensitive to boundary conditions and singular in the thermodynamical limit. Therefore, it cannot be directly used in any practical calculation.

It is obvious that the dipole operator still contains a regular contribution for interband transitions. To identify this contribution we have to work in momentum space and make use of eqs. (10) and (28a). Then we arrive at

$$
\begin{aligned}
\hat{P}_{\text {inter }}^{\mathrm{P}} & =\sum_{|k| \leq \pi /(2 a), \sigma} \mu_{\text {inter }}^{\mathrm{P}}(k)\left(\hat{a}_{k, \sigma,+}^{+} \hat{a}_{k, \sigma,-}-\hat{a}_{k, \sigma,-}^{+} \hat{a}_{k, \sigma,+}\right) \\
\mu_{\text {inter }}^{\mathrm{P}}(k) & =-\frac{i e a}{2}\left[\delta\left(\frac{2 t}{E(k)}\right)^{2}+\eta\right]
\end{aligned}
$$

for the interband dipole operator $(\operatorname{sgn}(\delta)=-\operatorname{sgn}(\eta))$. Note that it is impossible to give the expression for an "intraband dipole operator" because the $k$-th component of the intraband current operator $\hat{j}_{\text {intra }}^{\mathrm{P}}$ of eq. (28b) and the diagonalized Hamilton operator $\hat{T}(\delta)$ of eq. (10) are proportional to each other. In any case such an "intraband dipole operator" would not give a contribution to the optical (i.e., finite frequency) absorption, and can thus be ignored in the case of a Peierls insulator. 
In the form of equation (38) the interband dipole operator can equivalently well be used for the calculation of the optical and higher order susceptibilities for Peierls-distorted systems.

\section{SUMMARY}

In this paper we have presented a detailed analysis of the optical absorption in Peierls insulators. We observe the expected signature of a broad band-to-band transition with van-Hove singularities at the absorption edges. The strong momentum dependence of the transition matrix elements suppresses the singularity at the high-frequency edge compared to the one at the low-frequency edge. In contrast to previous investigations we gave explicit analytical expressions for the optical absorption and the dipole matrix elements for the tightbinding band structure. In addition we found correction (" $\eta$ "-)terms to the current operator and dipole matrix elements which have been ignored in previous works (Genkin and Mednis 1968); (Cojan, Agrawal, and Flytzanis 1977); (Baeriswyl, Harbeke, Kiess, Meier, and Meyer 1983); (Abe 1993). They arise because the distance between two neighboring sites is not constant but $\Delta r=a(1 \pm \eta / 2)$. However, they only slightly influence the final result for the optical absorption.

Unfortunately, a direct comparison to experiment is difficult for two reasons. First, disorder effects can inhomogeneously broaden single lines. Hence an experimentally observed band can very well be a sign of disorder rather than an argument for a Peierls insulator. Secondly, when a residual electron-electron interaction is included in a Peierls insulator (Abe, Yu, and Su 1992); (Abe, Schreiber, Su and Yu 1992) one can equally well obtain excitons which draw the oscillator strength form the band transitions. Hence, single exciton lines on the other hand are not a clear-cut indication against a Peierls insulator either. 


\section{ACKNOWLEDGMENTS}

We thank H. Bäßler, A. Horváth, M. Lindberg, S. Mazumdar, M. Schott, and G. Weiser for useful discussions. The project was supported in part by the Sonderforschungsbereich 383 "Unordnung in Festkörpern auf mesoskopischen Skalen" of the Deutsche Forschungsgemeinschaft.

\section{REFERENCES}

- S. Abe, in: Relaxation in Polymers, ed. by T. Kobayashi, (World Scientific, Singapore, (1993)), p. 215 .

- S. Abe, M. Schreiber, W. P. Su, and J. Yu, Phys. Rev. B 45, 9432 (1992).

- S. Abe, J. Yu, and W. P. Su, Phys. Rev. B 45, 8264 (1992).

- L. Alcácer, and A. Brau and J.-P. Farges, in: Organic Conductors, ed. by J.-P. Farges, (Marcel Dekker, New York, (1994)).

- D. Baeriswyl, G. Harbeke, H. Kiess, E. Meier, and W. Meyer, Physica B 117-118, $617(1983)$

- D. Baeriswyl, D. K. Campbell, and S. Mazumdar in Conjugated Conducting Polymers, ed. by H. Kiess, (Springer Series in Solid State Sciences 102, Springer, Berlin (1992)).

- C. Cojan, G. P. Agrawal, and C. Flytzanis, Phys. Rev. B 15, 909 (1977).

- J.-P. Farges (ed.), Organic Conductors, (Marcel Dekker, New York, (1994)).

- A. Fritsch and L. Ducasse, J. Physique I 1, 855 (1991).

- F. Gebhard, K. Bott, M. Scheidler, P. Thomas, and S.W. Koch, forthcoming articles.

- V. M. Genkin and P. M. Mednis, Zh. Eksp. Teor. Fiz. 54, 1137 (1968); (Sov. Phys. JETP 27, 609 (1968)). 
- H. Haug and S. W. Koch, Quantum Theory of the Optical and Electronic Properties of Semiconductors, (World Scientific, Singapore, (1990)).

- A. J. Heeger, S. Kivelson, J. R. Schrieffer, and W.-P. Su, Rev. Mod. Phys. 60, 781 (1988).

- G. D. Mahan, Many-Particle Physics, (2nd edition, Plenum Press, New York (1990)).

- P. F. Maldague, Phys. Rev. B 16, 2437 (1977).

- S. Mazumdar and S. N. Dixit, Phys. Rev. B 34, 3683 (1986).

- F. Mila, unpublished (1995).

- J. C. Philipps, Solid State Physics 18, ed. by F. Seitz, D. Turnbull, and H. Ehrenreich, 55 (1966).

- M. Schott and M. Nechtschein, in: Organic Conductors, ed. by J.-P. Farges, (Marcel Dekker, New York, (1994)). 


\section{FIGURES}

FIG. 1. Band structure of a Peierls insulator for $\delta=0.2$.

FIG. 2. Reduced optical conductivity, $\sigma_{\text {red }}(\omega>0)$ in a Peierls insulator for $\delta=0.2, \eta=-0.06$. A broadening of $\gamma=0.01 W$ has been included. 


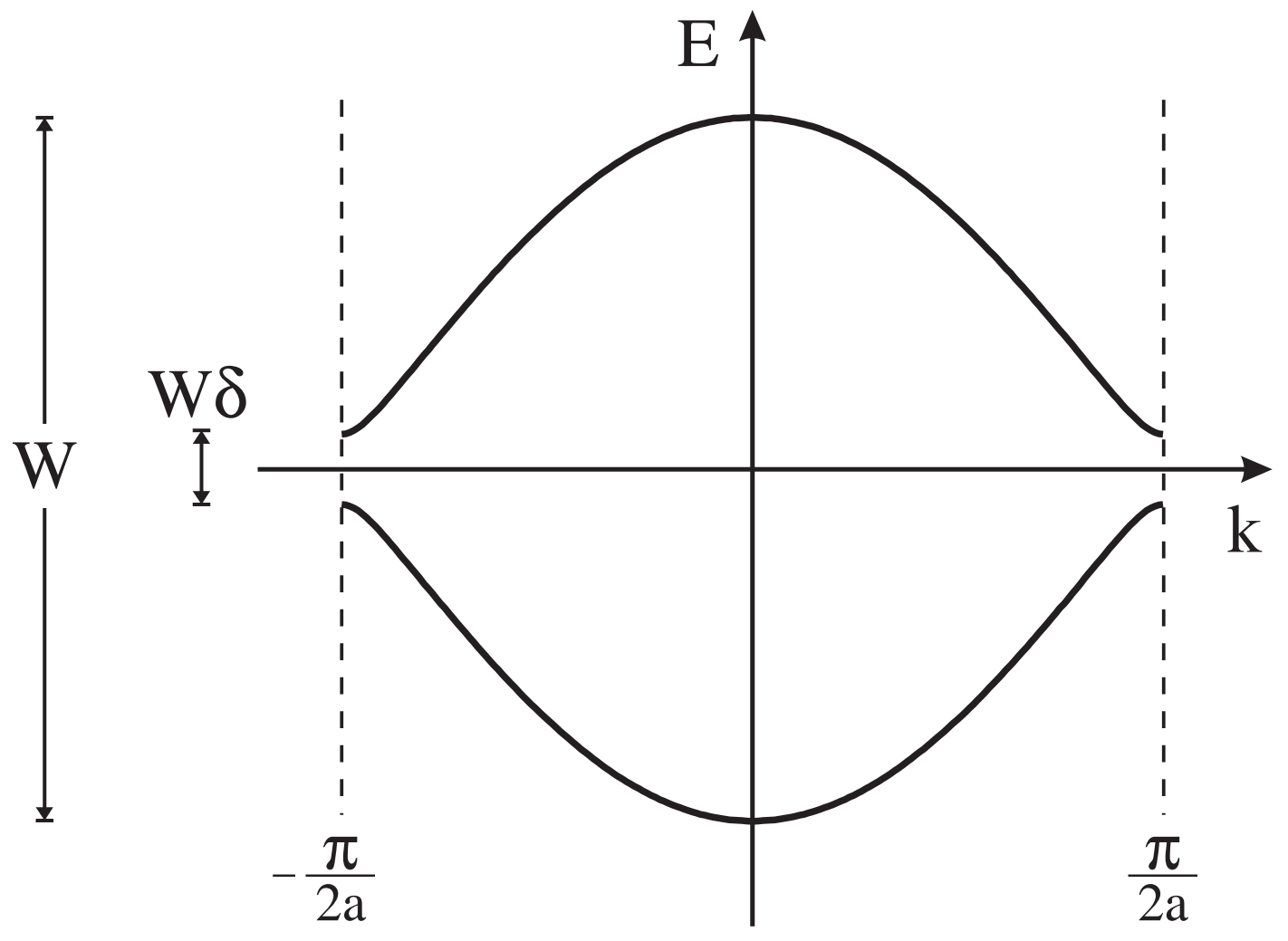

Fig. 1: F. Gebhard et al.,

Optical absorption of non-interacting tight-binding ... 


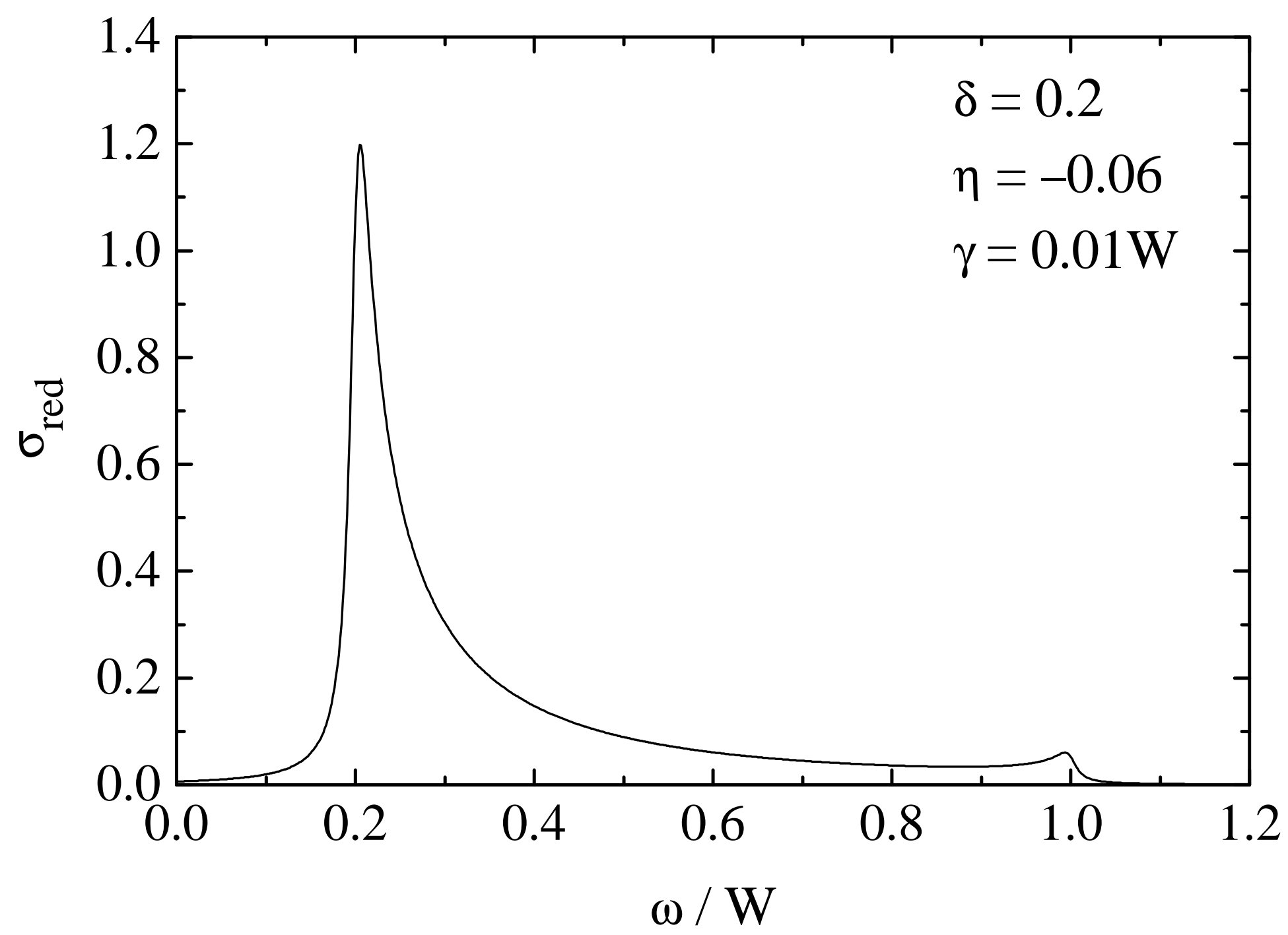

Fig. 2: F. Gebhard et al., Optical absorption of non-interacting tight-binding ... 